\title{
Recherche de la staphylocoagulase libre à partir du plasma d'animaux tropicaux : influence du type d'anticoagulant, de la température et de la durée de conservation
}

\section{Investigation of free staphylocoagulase from tropical animal plasma: influence of anticoagulant type, temperature and preservation's length}

\author{
Dougnon $\mathrm{V}^{* 1}$, Yehouenou $\mathrm{B}^{2}$, Dansi Soclo $\mathrm{F}^{1}$, Houefonde $\mathrm{A}^{1}$, Zehounkpe $\mathrm{F}^{1}$, Amadou $\mathrm{A}^{1}$, Aniambossou $\mathrm{A}^{1}$, \\ Fanou $\mathrm{B}^{1}$, Aclinou $\mathrm{E}^{2}$, Assogba $\mathrm{P}^{1}$, Fabiyi $\mathrm{K}^{1}$, Hounsa $\mathrm{E}^{1}$, DokpomiwaT ${ }^{1}$. \\ 1. Unité de Recherche en Microbiologie Appliquée et Pharmacologie des substances naturelles (U.R.M.A. \\ Pha), Ecole Polytechnique d'Abomey-Calavi (EPAC), Université d'Abomey-Calavi (UAC), 01 BP 2009 \\ Cotonou, Benin. \\ 2. Laboratoire de Biologie et Typage Moléculaire en Microbiologie (L.B.T.M.M) Département de \\ Biochimie et de Biologie cellulaire, Faculté des Sciences et Techniques (F.A.S.T), Université d'Abomey-Calavi \\ (UAC), 05 BP 1604 Cotonou, Benin. \\ Correspondance : DOUGNON Tamègnon Victorien, Laboratoire de Recherche en Biologie Appliquée, Ecole \\ Polytechnique d'Abomey-Calavi, Cotonou, Bénin, E-mail : urmaphaepac@hotmail.com
}

\section{RESUME}

L'identification biochimique de Staphylococcus aureus nécessite la recherche de la staphylocoagulase libre, réalisée grâce au plasma lyophilisé de lapin. Cette étude vise à promouvoir l'utilisation de plasma frais d'animaux tropicaux et déterminer l'influence des anticoagulants, de la température et de la durée de conservation de ces plasmas dans la révélation de la staphylocoagulase libre.Cinq (5) échantillons de sang de rats wistar, 3 de porc, 10 de poulets et 10 de lapins ont été recueillis sur anticoagulants. Après caractérisation biochimique d'une souche de référence de $S$. aureus ATCC 25923, la recherche de la staphylocoagulase libre a été faite à partir des plasmas d'animaux tropicaux conformément aux critères bactériologiques classiques. Des résultats obtenus, seuls les échantillons de plasma de lapins et de porcs ont permis la révélation de la staphylocoagulase libre. De tous les anticoagulants testés, l'EDTA et Citrate de sodium ont été les plus efficaces. Une part des plasmas de lapins recueillis sur EDTA a été conservée au réfrigérateur et l'autre part à température ambiante durant sept jours. La recherche de la staphylocoagulase a été faite tous les jours de conservation à partir des plasmas. Il découle de cette conservation que la température n'a pas d'influence majeure sur les échantillons de plasma sauf que leur conservation à température ambiante ralentit l'obtention des résultats. Il est donc préférable pour l'obtention de résultats dans un temps relativement court et pour éviter les risques de contamination des plasmas, de les conserver au réfrigérateur entre $2^{\circ}$ et $8^{\circ} \mathrm{C}$ pendant un maximum de 6 jours.

Mots clés: Staphylocoagulase libre, plasmas d'animaux tropicaux, S. aureus.

\section{Abstract}

biochemical identification of Staphylococcus aureus requires the search for free staphylocoagulase, performed using freeze-dried rabbit plasma. This study aims to determine the use of fresh tropical plasma and the influence of anticoagulants, temperature and shelf life of plasmas in the revelation of free staphylocoagulase. Five (5) blood samples from rats, 3 pork, 10 chickens and 10 rabbits were collected on anticoagulants. After biochemical characterization of the $S$. aureus ATCC 25923 reference strain, free staphylocoagulase was made from tropical animal plasmas. From the results, only plasma samples of rabbits and pigs allowed the disclosure of free staphylocoagulase. Of all the anticoagulants tested, EDTA and sodium citrate were the most effective. Part of rabbit plasma collected on EDTA was stored in the refrigerator and the other at room temperature for seven days. The search for staphylocoagulase was done every day from plasma. Results showed that temperature does not have a major influence on plasma samples, except that their conservation at room temperature slowed down the results. It is therefore preferable to obtain results in a relatively short time and to avoid the risks of contamination of the plasmas, to keep them in the refrigerator between $2^{\circ}$ and $8^{\circ} \mathrm{C}$ for a maximum of 6 days.

Key words: free Staphylocoagulase, tropical animal plasmas, S. aureus

\section{INTRODUCTION}

Les infections bactériennes constituent de nos jours un véritable problème sanitaire. Il existe un très grand nombre d'infections bactériennes pouvant affecter l'être humain. Si certaines infections bactériennes sont très bénignes, $d$ 'autres au contraire peuvent être mortelles. Dans la liste de ces bactéries, nous avons le genre Staphylocoque. Les staphylocoques sont des bactéries présentes dans notre environnement, au niveau de notre peau et de nos muqueuses. Ils sont constitués de plusieurs espèces dont Staphylococcus aureus qui occupe une place importante en pathologie humaine et animale $(1,2)$ Staphylococcus aureus est une bactérie ubiquiste et commensale de la peau et des voies nasales chez l'Homme (3). La capacité unique de $S$. aureus de provoquer la coagulation est connue depuis plus d'un siècle $(4,5)$. L'identification de $S$. aureus au laboratoire est donc basée, entre autres, sur la présence de la staphylocoagulase libre. Une 
divergence est notée dans la réalisation de la technique. La recherche de la staphylocoagulase libre doit être réalisée en tube à hémolyse en utilisant la suspension de plasma de lapin lyophilisé et le bouillon de $24 \mathrm{~h}$ à volume égal (6) Cependant, rare sont les laboratoires qui arrivent à le réaliser, compte-tenu du coût élevé du plasma lyophilisé de lapin utilisé dans le cadre de l'identification des souches de Staphylococcus aureus (7). Des travaux ont été entrepris pour montrer qu'il était possible de réduire le volume de plasma de lapin lyophilisé commercialisé et que le plasma frais de lapin pouvait être utilisé( 7,8$)$. Même si la preuve de l'efficacité du plasma frais de lapin a été apportée (7), qu'en est-il de l'influence des anticoagulants, de la température et de la durée de conservation sur la révélation de la staphylocoagulase libre avec un substituant au plasma lyophilisé de lapin ? C'est ce qui justifie cette étude dont l'objectif général est d'améliorer l'efficience du diagnostic bactériologique des infections à $S$. aureus. Spécifiquement, il s'est agi de : Mettre en évidence une staphylocoagulase libre éventuelle à partir des plasmas d'animaux tropicaux afin d'identifier le meilleur anticoagulant; Réaliser une étude comparative entre les résultats obtenus à partir des plasmas frais réfrigérés et ceux conservés à température ambiante au laboratoire,Déterminer la durée maximale de conservation du meilleur plasma frais.

\section{MATERIEL ET METHODES}

\section{MATERIEL}

Le matériel biologique utilisé dans le cadre de cette étude était composé de cinq (05) échantillons de sang de rats wistar, trois (03) de porc, dix (10) de poulets et dix (10) de lapins recueillis sur anticoagulants (EDTA, fluorure de sodium, citrate de sodium). Ces échantillons ont été recueillis chez des animaux en parfait état de santé dans une ferme d'élevage. Pour la caractérisation biochimique de la souche de référence de $S$. aureus ATCC 25923, les milieux de culture Chapman et Mueller Hinton ont été utilisés. La gélose Mueller Hinton a servi à réaliser le test de stérilité sur les échantillons de plasma et la recherche de la staphylocoagulase a été faite à partir du bouillon.

METHODES

Obtention et caractérisation de la souche de Staphylococcus aureus

La souche de référence ATCC 25923 a été obtenue au laboratoire de l'Unité de Recherche en Microbiologie Appliquée et Pharmacologie des substances naturelles (U.R.M.A.Pha). La caractérisation de cette dernière a été faite le jour même selon les normes standards du diagnostic biologique de Staphylococcus aureus en laboratoire de bactériologie. L'état frais, l'état coloré et l'ensemencement sur les milieux de Chapman et de Mueller Hinton ont donc été faits. La lecture des géloses ensemencées a été faite le $2^{\text {ème }}$ jour et des tests biochimiques ont été réalisés. La recherche de la staphylocoagulase libre a été réalisée sur le bouillon Muller Hinton de 24 heures ensemencé et du plasma lyophilisé de lapin commercialisé. Dans un tube à hémolyse, $0,5 \mathrm{ml}$ de plasma lyophilisé de lapin a été ajouté à $0,5 \mathrm{ml}$ du bouillon de 24 heures ensemencé avec la souche de référence ATCC 25923. Un témoin négatif a été préparé en mélangeant le bouillon non ensemencé au plasma lyophilisé de lapin. Le mélange a été incubé à l'étuve à $37^{\circ} \mathrm{C}$.

Prélèvement des animaux et obtention des échantillons de plasma frais

Le $3^{\text {ème }}$ jour, les rats Wistar ont été prélevés par ponction rétro-orbitale, les poulets ont été prélevés au niveau de la veine alaire, et les lapins et les porcs au niveau de la veine marginale de l'oreille. Les échantillons de sang de lapin et de porc ont été recueillis dans des tubes EDTA, Citrate de sodium et Fluorure de Sodium et ceux de rats Wistar et de poulets ont été recueillis uniquement sur EDTA puis le tout a été centrifugé à 1500 tours $/ \mathrm{min}$ pendant $15 \mathrm{~min}$. Le plasma recueilli au niveau de chaque échantillon a été décanté dans des tubes Eppendorf. A partir de ces plasmas, a été réalisé un test de stérilité en les ensemençant sur gélose Muller Hinton.

\section{Effet des anticoagulants}

On a mélangé $0,5 \mathrm{ml}$ du bouillon de cet isolat à $0,5 \mathrm{ml}$ de plasmas frais de lapin et de porc respectivement sur EDTA, Fluorure de sodium et Citrate de sodium et la lecture des résultats a été faite à $1 \mathrm{~h}, 2 \mathrm{~h}, 4 \mathrm{~h}, 6 \mathrm{~h}$ et $24 \mathrm{~h}$.

Comparaison de l'efficacité des échantillons de plasma frais

Le $4^{\text {ème }}$ jour, la réalisation de la staphylocoagulase libre a été faite à partir des différents plasmas d'animaux tropicaux dans le but de déterminer un substituant au plasma lyophilisé de lapin et l'anticoagulant le plus efficace pour la révélation de la staphylocoagulase libre.

Monitorage de la durée de conservation du meilleur plasma

Une portion des meilleurs échantillons de plasma retenus, a été conservée au réfrigérateur entre $2^{\circ} \mathrm{C}$ et $8^{\circ} \mathrm{C}$ et l'autre portion a été conservée à température ambiante du laboratoire pendant 7 jours. La recherche de la staphylocoagulase libre a été faite à partir de ces deux portions de plasma durant les 7 jours de conservation selon les normes usuelles en laboratoire de bactériologie $(6,9)$.

\section{RESULTATS}

\section{Effet des anticoagulants}

L'analyse du tableau I montre que 90\% des échantillons de plasma de lapin recueillis sur EDTA ont donné un résultat positif à la recherche de la staphylococulase libre pour la souche de Staphylococcus aureus ATCC 25923 tandis que $100 \%$ des échantillons de plasma recueillis sur Fluorure de sodium et Citrate de sodium ont donné 
un résultat positif avec la même souche. $100 \%$ des échantillons de plasma de porcs (03) recueillis sur EDTA, sur Fluorure de sodium et sur Citrate de sodium ont donné un résultat positif à la recherche de la staphylococulase libre pour la souche de Staphylococcus aureus ATCC 25923.(Tableau II). Il ressort de l'analyse du tableau III qu'à 1h, 9/10 échantillons de plasma de lapin recueilli sur EDTA et $9 / 10$ recueilli citrate de sodium se sont révélés positifs mais aucun des échantillons recueillis sur Fluorure de sodium n'a coagulé. Au bout de 2h, 9/10 échantillons de plasma recueilli sur EDTA et 10/10 des échantillons de plasma sur citrate de sodium sont positifs mais toujours aucun des échantillons de plasma fluorure de sodium n'a coagulé. A 24h, 9/10 échantillons de plasma recueilli sur EDTA, 10/10 de Citrate de Sodium et 10/10 de Fluorure de sodium. Du tableau IV, on remarque que les échantillons de plasmas de porcs recueillis sur les trois anticoagulants n'ont réagi qu'au bout de $24 \mathrm{~h}$.

Effet de la température et de la durée de conservation

La présente étude révèle que les échantillons de plasma de poulets testés ne sont coagulés qu'au bout de $24 \mathrm{~h}$ et ce dans une faible proportion (30\%) tandis que tous les échantillons de plasmas de lapins sont coagulés au bout d'une heure. De l'étude de la révélation de la staphylocoagulase avec les échantillons de plasma de rongeurs, il ressort que le plasma de Rat Wistar ne donne pas de bons résultats. En effet au bout de $24 \mathrm{~h}$, seulement $20 \%$ des échantillons de plasma de rat Wistar ont coagulé alors qu'au bout d'une heure déjà ceux de lapin l'étaient (Figure 1). Les résultats obtenus avec les échantillons de plasma de poulets et de rats Wistar n'étant pas probant, l'expérimentation a été poursuivie uniquement avec les plasmas de lapins. Durant les trois premiers jours d'essai, il a été constaté que tous les échantillons de plasmas de lapins ont permis la révélation de la staphylocoagulase libre que ce soit à température ambiante ou au réfrigérateur (figure2). $\mathrm{Du} 4^{\mathrm{e}}$ au $6^{\mathrm{e}}$ jour de conservation, on note la coagulation de tous les échantillons de plasma réfrigérés la première heure d'incubation tandis que celle des plasmas laissés à température ambiante connait un retard d'une heure (figure 3 ). Au $7^{\mathrm{e}}$ jour d'essai, en $24 \mathrm{~h}$ seulement $50 \%$ des échantillons de plasma de lapins réfrigérés sont coagulés contre $20 \%$ de ceux conservés à température ambiante (figure 4)

\section{COMMENTAIRE}

Cette étude a visée analytique a eu pour objectif général, d'améliorer le diagnostic biologique des staphylococcies. Ainsi, pour la validation des résultats, le test à la staphylocoagulase libre avec le plasma lyophilisé du lapin sur la souche Staphylococcus aureus de référence ATCC25923 a révélé qu'elle est productrice de coagulase libre. Le bouillon de cet isolat a coagulé $90 \%$ échantillons de plasma de lapin recueilli sur EDTA de résultat positif en deux heures pour la recherche de la staphylococulase libre pour la souche de Staphylococcus aureus ATCC 25923. Ce même bouillon a coagulé $100 \%$ échantillons de plasma de lapin recueilli sur Citrate de sodium de résultat positif en deux heures tandis que tous les dix échantillons de plasma recueilli sur Fluorure de sodium ont coagulé soit $100 \%$ de résultat positif avec la même souche en vingt-quatre heures. On a $100 \%$ de résultat positif avec les échantillons de plasma frais de porc (03) recueillis sur les mêmes anticoagulants en vingt-quatre heures (24h). En réponse à la privation de fer, $S$. aureus produit de la staphyloferrine $B$, un sidérophore contenant du citrate qui refoule le fer vers la cellule(10). Ainsi la présence de citrate chez $S$. aureus pourrait entrainer de réaction de coagulase faussement positive avec le plasma recueilli sur Citrate de sodium mais étant donné que le même plasma recueilli sur EDTA et Fluorure de sodium a donné un résultat positif, cette éventualité de « faux positif » avec le citrate de sodium peut être éliminée. L'EDTA n'est pas utilisé par les bactéries, ce qui n'entraînera pas de réaction de coagulase faussement positive par effet des bactéries qui utilisent le citrate (11). La révélation de la staphylocoagulase libre avec le tube Fluorure de sodium chez les lapins a été lente, mais ceci n'a pas d'influence sur la qualité des résultats dans un temps d'incubation élargi. Au regard des résultats obtenus, les échantillons de plasma de poulets ne constituent pas un bon candidat pour la révélation de la staphylocoagulase libre $(30 \%$ de plasmas coagulés en 24h). Par contre, les échantillons de plasma de lapins sont d'une efficacité accrue dans la révélation de cette enzyme (100\% de plasmas de lapins coagulés au bout d'une heure de temps). Ces résultats corroborent ceux de Adesiyun et Shehu.(12). Etant donné que le mécanisme de coagulation implique la conversion $\mathrm{du}$ fibrinogène en fibrine par un complexe enzymatique, constitué de coagulase plus le facteur de réaction à la coagulase ou activateur de coagulase (13), ces différences observées seraient dues au fait que les échantillons de plasma de lapins sont plus concentrés en facteur de réaction à la coagulase que les échantillons de plasma de poulets. Les échantillons de plasma de rats Wistar ont donné $20 \%$ de réussite. Ce faible taux de réussite du plasma de rats peut traduire la non spécificité et la non fiabilité du plasma en présence de la souche ATCC 25923 (14). Le plasma de porc est efficace mais celui du lapin est plus efficient. L'étude sur l'effet de la température et de la conservation a donc été faite avec le plasma le plus efficient. Des essais réalisés sur les échantillons de plasma de lapins réfrigérés et conservés à température ambiante, on note que durant les trois premiers jours de conservation, $100 \%$ des plasmas 
testés (plasmas réfrigérés comme plasmas laissés à température ambiante) ont révélé la staphylocoagulase positive au bout d'une heure de temps. $\mathrm{Du} 4^{\mathrm{e}}$ au $6^{\mathrm{e}}$ jour d'expérimentation avec les échantillons de plasma de lapins laissés à température ambiante, on note un retard d'une heure dans la révélation de la staphylocoagulase libre $(00 \%$ de résultat positif à $1 \mathrm{~h}$ d'incubation) tandis que tous les échantillons de plasma réfrigérés réagissent au bout d'une heure. Il ressort des essais $\mathrm{du} 7^{\mathrm{e}}$ jour de conservation qu'en $24 \mathrm{~h}, 50 \%$ des plasmas de lapins réfrigérés de lapins testés mettent en évidence la staphylocoagulase libre contre $20 \%$ des plasmas laissés à température ambiante. Néanmoins, l'influence de cette altération subie par le plasma frais conservé n'a été remarquée qu'au bout de six jours contrairement au constat fait par Ibrahim en 2014 qui rapportait une altération au bout de 5 jours. Ces résultats pourraient s'expliquer par le fait que la conservation à température ambiante à un effet dénaturant sur les échantillons de plasma frais de lapins et ralentissant dans l'obtention des résultats. Les résultats du présent travail nous amènent à dire que les échantillons de plasma frais de lapins pourraient substituer valablement le REFERENCES

1. Boerlin P, Kuhnert P, Hüssy D, Schaellibaum M. Methods for identification of Staphylococcus aureus isolates in cases of bovine mastitis. J Clin Microbiol. févr 2003;41(2):767-71.

2. Weist K. Evaluation of six agglutination tests for Staphylococcus aureus identification depending upon local prevalence of meticillin-resistant S. aureus (MRSA). J Med Microbiol. 1 mars 2006;55(3):283-90.

3. Kateete DP, Kimani CN, Katabazi FA, Okeng A, Okee MS, Nanteza A, et al. Identification of Staphylococcus aureus: DNase and Mannitol salt agar improve the efficiency of the tube coagulase test. Ann Clin Microbiol Antimicrob. 13 août 2010;9:23.

4. Peetermans M, Vanassche T, Liesenborghs L, Claes J, Vande Velde G, Kwiecinksi J, et al. Plasminogen activation by staphylokinase enhances local spreading of S. aureus in skin infections. BMC Microbiol [Internet]. déc 2014 : http://bmcmicrobiol.biomedcentral.com/articles/10.1186/s12866-014-0310-7

5. Waters EM, Rowe SE, O'Gara JP, Conlon BP. Convergence of Staphylococcus aureus Persister and Biofilm Research: Can Biofilms Be Defined as Communities of Adherent Persister Cells? Kline KA, éditeur. PLOS Pathog. 29 déc 2016;12(12):e1006012.

6. Carbonnelle B. Bactériologie médicale: techniques usuelles. Paris: Simep; 1990.

7. Bankole H., Dougnon T., Fiogbé E., Amoussou A., Baba-moussa L. Identification de Staphylococcus aureus : est-il possible de réduire le volume de plasma de lapin sans influencer la recherche de la staphylocoagulase libre? Journal of Applied Biosciences. 73:6027-6032.

8. Ibrahim B. Identification de staphylococcus aureus : utilisation du plasma frais de lapin pour la recherche de la staphylocoagulase libre [Mémoire pour l'obtention du diplôme de licence professionnelle en Analyses Biomédicales]. Ecole Polytechnique d'Abomey-Calavi, Université d'Abomey-Calavi;

9. Hanane A. Isolement des souches de Staphylococcus aureus résistantes à la méthicilline: Etude de leur sensibilité aux autres familles d'antibiotiques [Mémoire pour l'obtention du diplôme de master en microbiologie appliqué et biotechnologie microbienne]. Faculté Des Sciences de la Nature et de la Vie. Université Mentouri, Constantine, Algérie;

10. Kobylarz MJ, Grigg JC, Sheldon JR, Heinrichs DE, Murphy MEP. SbnG, a citrate synthase in Staphylococcus aureus: a new fold on an old enzyme. J Biol Chem. 5 déc 2014;289(49):33797-807.

11. Buddingh GJ. Bergey's Manual of Determinative Bacteriology: 8th edition, edited by R. E. Buchanan and N. E. Gibbons. xxvii $+1,246$ pages, illustrated. The Williams and Wilkins Company, Baltimore, Maryland. 1974. No price. Am J Trop Med Hyg. 1 mai 1975;24(3):550-550.

12. Adesiyun AA, Shehu LM. Detection of staphylo-coagulase using plasmas from various animals. Vet Microbiol. juin 1985;10(4):387-92.

13. Lorenz LL, Duthie ES. Staphylococcal Coagulase: Mode of Action and Antigenicity. Microbiology. 1 févr 1952;6(1-2):95-107. 
14. Mohamed E. «Conventional and molecular epidemiology of Staphylococcus aureus isolates originated from human animals » a thesis submitted in partial Fulfilment of Master Degree of Veterinary Science Faculty of Veterinary Medecine. University of Khartoum; 2003.

Tableau I : Répartition des résultats de la coagulase libre obtenus avec des colonies de $24 \mathrm{~h}$ émulsionnées au plasma frais de lapin.
Résultats
EDTA
Fluorure de sodium
Citrate de sodium

\begin{tabular}{|c|c|c|c|}
\hline $\begin{array}{c}\text { Nombre de plasma ayant } \\
\text { coagulé }\end{array}$ & 9/10 & $10 / 10$ & $10 / 10$ \\
\hline Pourcentage & $90 \%$ & $100 \%$ & $100 \%$ \\
\hline
\end{tabular}

Tableau II : Répartition des résultats de la coagulase libre obtenus avec des colonies de 24 h émulsionnées au plasma frais de porc
Résultats
EDTA
Fluorure de sodium
Citrate de sodium

\begin{tabular}{|c|c|c|c|}
\hline $\begin{array}{c}\text { Nombre de plasma ayant } \\
\text { coagulé }\end{array}$ & $3 / 3$ & $3 / 3$ & $3 / 3$ \\
\hline Pourcentage & $100 \%$ & $100 \%$ & $100 \%$ \\
\hline
\end{tabular}

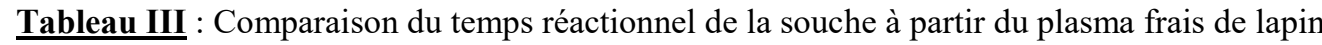

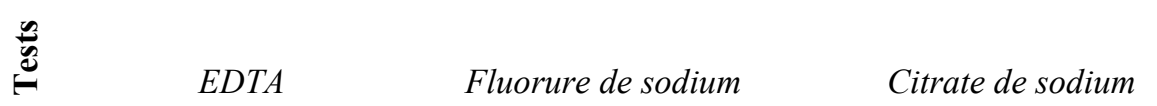

Temps

\begin{tabular}{cccc}
\hline \hline $1 h$ & 9 & 0 & 9 \\
$2 h$ & 9 & 0 & 10 \\
$4 h$ & 9 & 0 & 10 \\
$6 h$ & 9 & 0 & 10 \\
$24 h$ & 9 & 10 & 10 \\
\hline
\end{tabular}




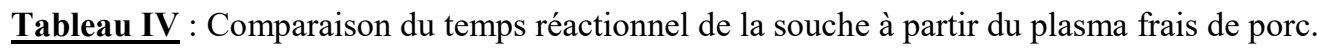

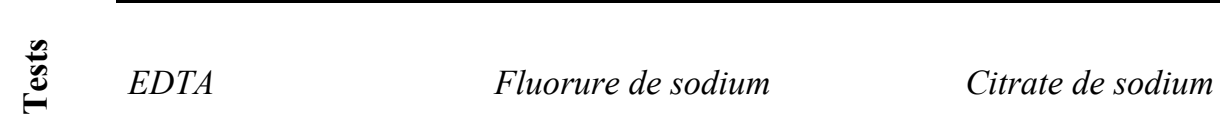

Temps

\begin{tabular}{llll}
\hline \hline $1 h$ & 0 & 0 & 0 \\
$2 h$ & 0 & 0 & 0 \\
$4 h$ & 0 & 0 & 0 \\
$6 h$ & 0 & 0 & 0 \\
$24 h$ & 3 & 3 & 3 \\
\hline
\end{tabular}

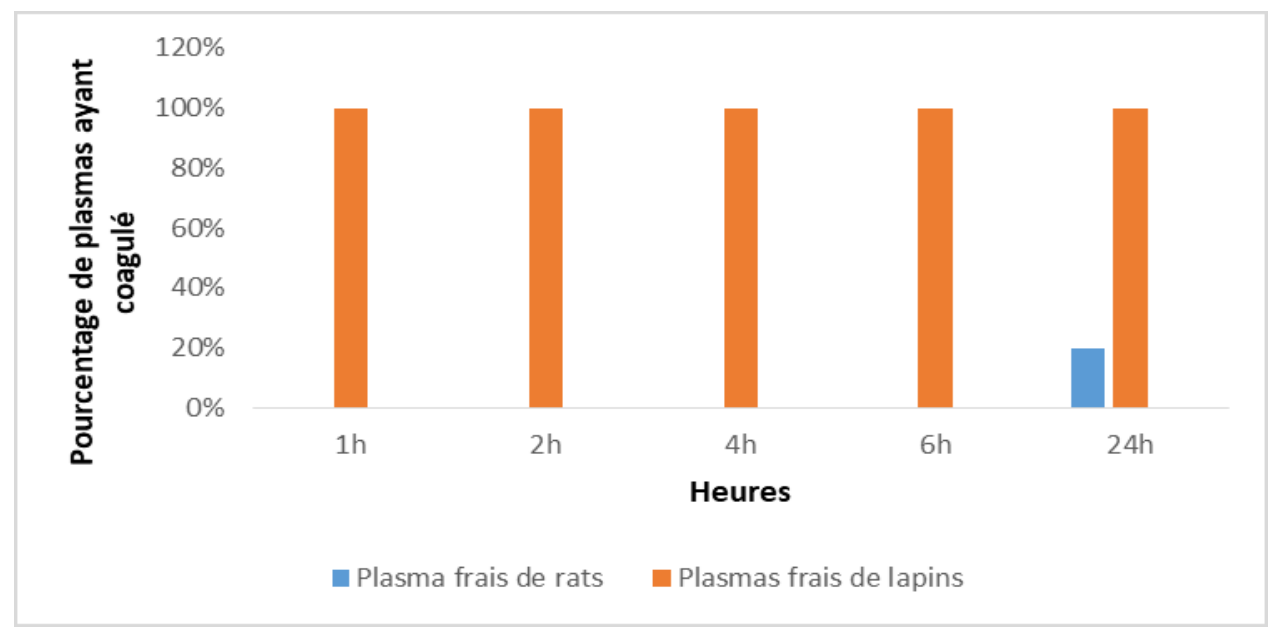

Figure 1: Comparaison de l'efficacité des plasmas frais de lapin et de rats Wistar selon le temps

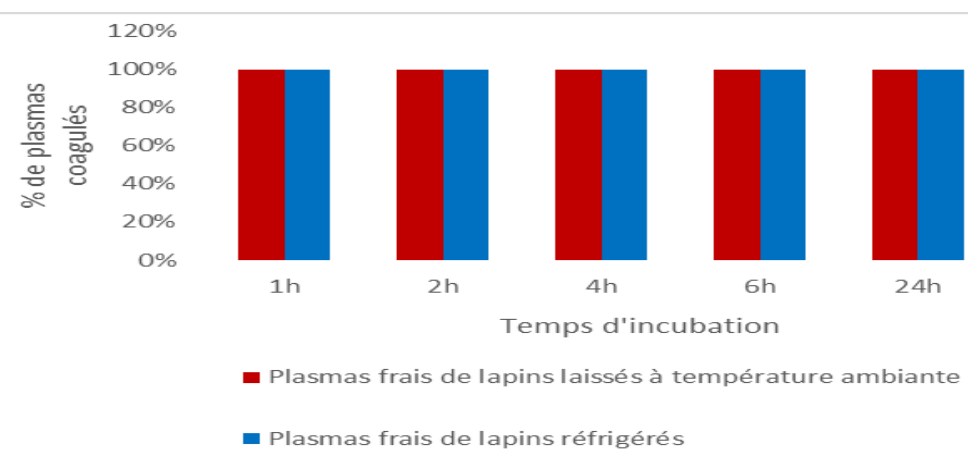

$\underline{\text { Figure 2 }}$ : Performance des plasmas frais de lapins au cours des trois premiers jours de 
conservation.

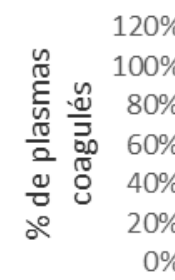

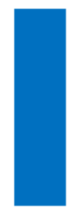

$1 \mathrm{~h}$

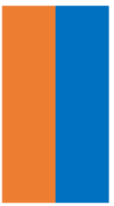

$2 \mathrm{~h}$

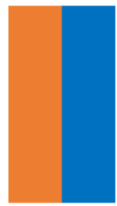

$4 \mathrm{~h}$

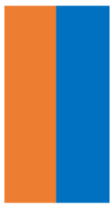

$6 \mathrm{~h}$

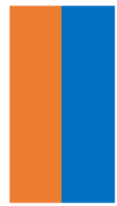

$24 \mathrm{~h}$

Temps d'incubation

— Plasmas frais de lapins laissés à température ambiante

- Plasmas frais de lapins réfrigérés

$\underline{\text { Figure 3 }}$ : Mise en évidence de la staphylocoagulase libre du $4^{\mathrm{e}}$ au $6^{\mathrm{e}}$ jour de conservation avec du plasma frais de lapins

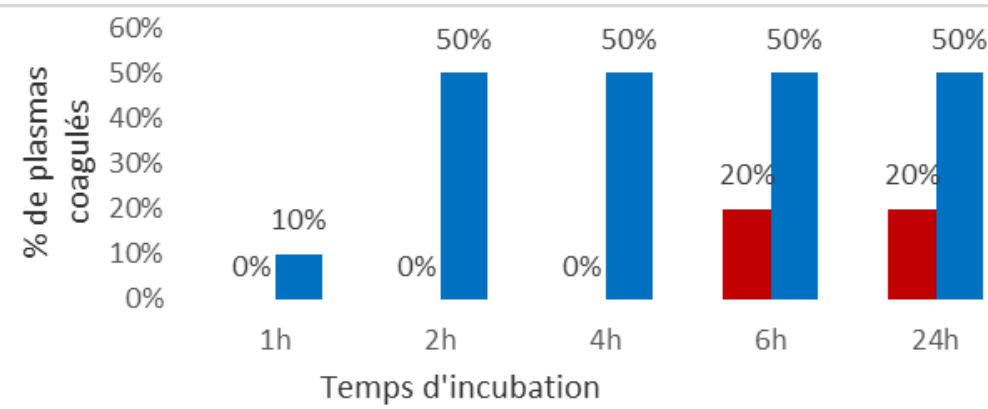

- Plasmas frais de lapins laissés à température ambiante

- Plasmas frais de lapins réfrigérés

$\underline{\text { Figure 4 }}$ : Révélation de la staphylocoagulase libre au $7^{\mathrm{e}}$ jour de conservation avec du plasma frais de lapins 